\title{
Prevention of Rheumatic Fever by Continuous Photodynamic Therapeutic
}

\author{
Kate C Blanco*, Jennifer M Soares, Natalia M Inada and Vanderlei S Bagnato \\ São Carlos Institute of Physics, Brazil
}

*Corresponding author: Kate C Blanco, São Carlos Institute of Physics, Brazil

\begin{tabular}{l} 
ARTICLE INFO \\
Received: 幽 May 29, 2019 \\
Published: 冓 June 07, 2019 \\
\hline Citation: Kate C Blanco, Jennifer M \\
Soares, Natalia M Inada, Vanderlei S Ba- \\
gnato. Prevention of Rheumatic Fever \\
by Continuous Photodynamic Therapeu- \\
tic. Biomed J Sci \& Tech Res 18(4)-2019. \\
BJSTR. MS.ID.003190.
\end{tabular}

Abbreviations: PT: Post Pharyngotonsillitis; RF: Rheumatic Fever; WHO: World Health Organization; CR: Rheumatic Carditis; WHF: World Heart Federation

\begin{abstract}
Introduction: Non and suppurative complications such as rheumatic fever and poststreptococcal acute glomerulonephritis may occur post-pharyngotonsillitis (PT). The conventional treatment of bacterial PT consists of orally administered antibiotics. Rheumatic fever (RF) incidence in developing countries with high prevalence PT are significant public health concerns. The diagnosis is imprecise due to difficulty of detecting the etiologic agent by laboratory methods in some countries. Associated with this, the dissemination of bacteria resistance to multiple antimicrobial agents to secondary prophylaxis by PDT was analyzed in cases of PT complications.
\end{abstract}

Case Report: A 37-year-old woman was clinically diagnosed with recurrent streptococcal PT. The second episode of PT associated to asymmetric arthritis and the results of lab tests lead to the diagnosis of rheumatic fever. A PDT session was performed every 30 days as secondary prophylaxis. Conclusion: This case hypothesis that oropharyngeal PDT may be an effective prophylactic treatment of rheumatic fever. However, more research is needed to test this theory.

\section{Introduction}

The main cause of PT is Streptococcus pyogenes, Lancefield group a beta hemolytic streptococcus (EBHGA) Ba -Saddik et al. The relative frequency of microorganisms depends on the age, geographic region and season in which they occurred Principi et al. The clinical diagnosis of Streptococcal PT can promote correct treatment and prevention of complications. However, there is no clinical sign or symptom that accurately identifies this bacterial infection. The etiological diagnosis depends on the detecting the agent by lab methods. Clinical scoring systems can be used as a reference to minimize the risk of EBHGA infection in places poor living conditions. Acute PT represents a source of social disorders and repeated use of antibiotics way [1]. It presents complications such as peritonsillar and retropharyngeal abscess, otitis, sinusitis, pneumonia, rheumatic fever and poststreptococcal glomerulonephritis. According to the World Health Organization (WHO) new cases of PT (around 600 million) occur due to EBHGA which 500 thousand can progress to RF about 300 thousand rheumatic carditis (CR). International organization such as the
World Heart Federation (WHF), the WHO and the African Union have promoted scientific measures to eradicate these diseases.

A GAS infection does not need to be symptomatic disease to trigger a recurrence. However, prevention of disease recurrence requires continuous antimicrobial prophylaxis. Secondary prevention of rheumatic fever is performed with prophylaxis initiated as soon as the disease is diagnosed. A complete antibiotic cycle should be administered in order to treat acute rheumatic and eradicates residual GAS. RF is an inflammatory disease and results from an autoimmune response to infection caused by GAS (CARAPETIS; MCDONALD; WILSON, 2005). Monthly doses of penicillin for rheumatic fever treatment has been approved for carditis (10 years) and RF (5 years). Photodynamic antimicrobial therapy is a noninvasive therapeutic modality for treatment of infectious diseases caused by different bacterial species [2]. Oxygen-Dependent Photochemistry reaction occurs in response to the activation of PS by a light source leading to the generation of cytotoxic reactive oxygen species. PDT has been effective and safe for use in the oral cavity for both dental and oral disinfection. 


\section{Case Presentation}

A 37-year-old woman was fine until August 2016, when she developed difficulty swallowing, sore throat, fever of $39^{\circ} \mathrm{C}$ and chills. She sought the hospital emergency service 15 days after symptom initiation which was diagnosed clinically with streptococcal PT. It was treated with levofloxacin hemihydrate 500mg. A new episode of PT occurred after seven days of the onset of first case which was associated with arthritis knees and shoulder. The condition evolved to partial improvement of pain, maintaining right shoulder pain. The results of the tests showed ASO (antistreptolysin 0 antibody) $997 \mathrm{IU} / \mathrm{mL}$ and VSH (blood staining speed) $37 \mathrm{~mm} / \mathrm{h}$ and PCR (C-reactive protein) $0.62 \mathrm{mg} / \mathrm{dL}$. Taking the hypothesis of asymmetric polyarthritis of large joints from rheumatic fever as main hypothesis [3]. The patient was advised to keep levofloxacin $500 \mathrm{mg}$ for another seven days. A PDT session was performed every three weeks. The patient chewed a curcumin gum (PDT Pharma) $22.5 \mathrm{mg} /$ united about 2 minutes, and the oral cavity was illuminated with a blue light emitting system (Patent: BR10201601347) at 450 $\mathrm{nm}, 20 \mathrm{~mW} / \mathrm{cm}^{2}$ and $2.4 \mathrm{~J} / \mathrm{cm}^{2}$.

\section{Discussion}

Difficulty swallowing, sore throat, fever of $39^{\circ} \mathrm{C}$ are common signs of streptococcal PT. The disease usually is manifested around 7 to 15 days after an infectious episode of PT. Careful anamnesis and physical examination are the diagnostic evaluation of the patient with suspected of PT in Brazil. Brazilian hospitals don't have access to tests of Beta-hemolytic Group A Streptococcus for suspicious cases. Recurrent PT disease occurred when the patient who were previously treated develop a new disease episode after seven days. This case was associated with asymmetric arthritis due to Rheumatic fever [4]. Rheumatic fever is a complication of this infection which affect various parts of the body, such as joints, heart, nervous system and skin. The diagnosis of PT increases their sensitivity depending on the disease target (CARAPETIS; MCDONALD; WILSON, 2005). In general, Brazil's public health service are limited, and when cases of acute and recurrent sore throat are managed in hospital assistance programs unrealized laboratory diagnostics. The symptoms of streptococcal PT and RF may overlap and it share the same treatments. The clinical manifestation studied here is the most common manifestation of $\mathrm{RF}$ [5]. It is characterized by an inflammation of the joints. It usually occurs one to three weeks after infection by the microorganism. In this clinical case study of RF was manifested 22 days from the beginning of PT infection. Guide to Clinical diagnosis of rheumatic fever is based on previous laboratory evidence of GAS infection with increased at least twice the title streptococcal serum samples from between 14 and 28 days apart.

The measured ASO is an antibody directed against streptolysin O, a toxic enzyme produced by bacteria of group A Streptococcus. Symptoms of rheumatic fever and high ASO level of $997 \mathrm{IU} / \mathrm{mL}$ aided in confirming the diagnosis. Elevated antibody titer means that, likely to have had a recent infection caused by streptococci.
However, the ASO test doesn't predict whether complications occurred after a streptococcal infection. VSH is considered by many to be a valuable aid in the RF prognosis. In acute inflammations VSH increases with the leucocyte count. Patients already developed rheumatic fever with infective endocarditis or joint infection require continued antibiotic prophylaxis to prevent relapse of the disease. The scientific justification for this prophylaxis is the elimination or reduction of transient bacteremia. The use of antibiotic though effective against the pathogen also act on bacteria beneficial to the human body, such as those present on the intestinal flora. The imbalance of the natural microbiota may result in the prevalence of opportunistic microorganisms or the artificial selection of bacteria resistant to antibiotics.

The patient who take antibiotic may present symptoms as stomach pains due to irritate the tissue with long periods of medication. In addition to these side effects of prolonged use of antibiotics. In 2050 the predicted deaths caused by resistant bacteria will be 10 million, as case numbers increase each year while the number of new antibiotics don't have the same evolution. For this reason, it is necessary to study new techniques that may be efficient against microorganisms, has little or no side effects to the patient and act for bacterium doesn't acquire resistance to the mechanism of action. These are some of the benefits of using PDT. PDT can be used from the initial stage of PT to avoid the progression to RF. It acts directly on Streptococcus sp. bacteria to minimize interaction with $\mathrm{T}$ cells. In this case reported, since the number of bacteria can be reduced by PDT and there are chance of causing reduced $\mathrm{RF}$ after recurrent PT.

\section{Conclusion}

In conclusion, based on the experience of this case report and a review of the current specialist literature, we advise that PDT, when performing secondary prophylaxis in patients with episode of rheumatic fever, consider a trial of oral treatment and report their findings.

\section{Acknowledgment}

This work has been supported in part by FAPESP (CEPID/ CEPOF 13/07276-1).

\section{References}

1. Goyal P, Vijayvergiya R (2016) Rheumatic Fever and Rheumatic Heart Disease. International Encyclopedia of Public Health 357-362.

2. O'neill J (2016) Tackling drug-resistant infections globally: final report and recommendations. Review on Antimicrobial Resistance.

3. Purchiaroni F, Tortora A, Gabrielli M, Bertucci F, Gigante G, et al. (2013) The role of intestinal microbiota and the immune system. European review for medical and pharmacological sciences 17(3): 323-333.

4. Von Rosenvinge EC, Song Y, White JR, Maddox C, Blanchard T, et al. (2013) Immune status, antibiotic medication and $\mathrm{pH}$ are associated with changes in the stomach fluid microbiota. ISME Journal 7(7): 1354-1366.

5. Wainwright M, Maisch T, Nonell S, Plaetzer K, Almeida A, et al. (2017) Photoantimicrobials are we afraid of the light? The Lancet Infectious Diseases 17(2): 49-55. 


\section{ISSN: 2574-1241}

DOI: 10.26717/BJSTR.2019.18.003190

Kate C Blanco. Biomed J Sci \& Tech Res

(c) (P) This work is licensed under Creative

Submission Link: https://biomedres.us/submit-manuscript.php

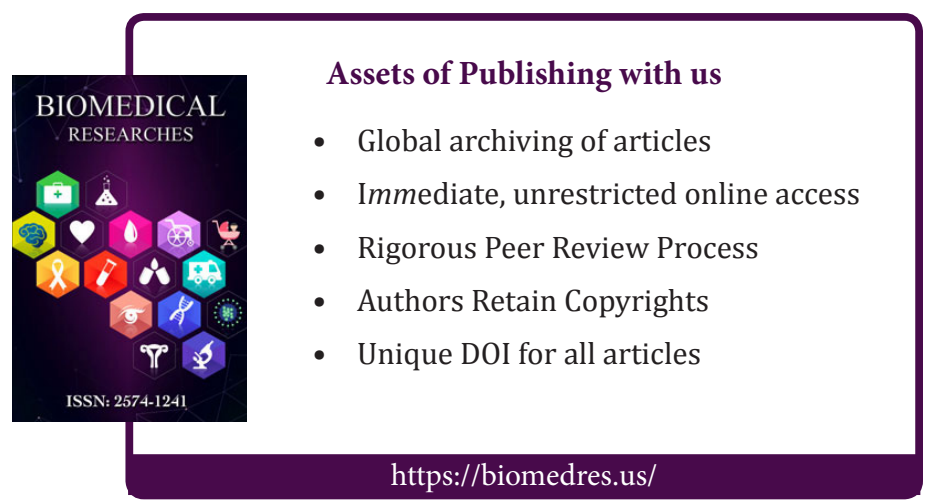

